\title{
Morpheus Lander Roll Control System and Wind Modeling Extended Abstract
}

\author{
Elisabeth A. Gambone \\ NASA Johnson Space Center, Houston, Texas, 77058 \\ 281-483-8285 \\ Elisabeth.A.Gambone@nasa.gov
}

The Morpheus prototype lander is a vertical testbed developed by NASA Johnson Space Center to assess advanced technologies. Between December 2013 and May 2014, Morpheus completed a series of flight tests at Kennedy Space Center (KSC) to demonstrate autonomous landing and hazard avoidance for future exploration missions. The vehicle is fueled with liquid oxygen and liquid methane, a clean and non-toxic propellant. As a prototype vehicle being tested in Earth's atmosphere, Morpheus requires a robust roll control system (RCS) to counteract aerodynamic forces. This paper describes the control algorithm designed that requests firing and delay times based on roll orientation. Design, analysis, and testing are supported using a high-fidelity simulation of vehicle dynamics. This paper also details the wind profiles generated using historical wind data, which are necessary to validate the RCS in the simulation environment. In preparation for Morpheus testing, the wind model was expanded to create day-of-flight (DOF) wind profiles based on data delivered by the KSC meteorology group.

The Morpheus RCS consists of four thrusters fueled by the main engine propulsion tanks. Two thrusters in both the positive and negative roll direction maintain vehicle pointing during flight. The thrusters are oriented on the vehicle as shown in Figure 1.

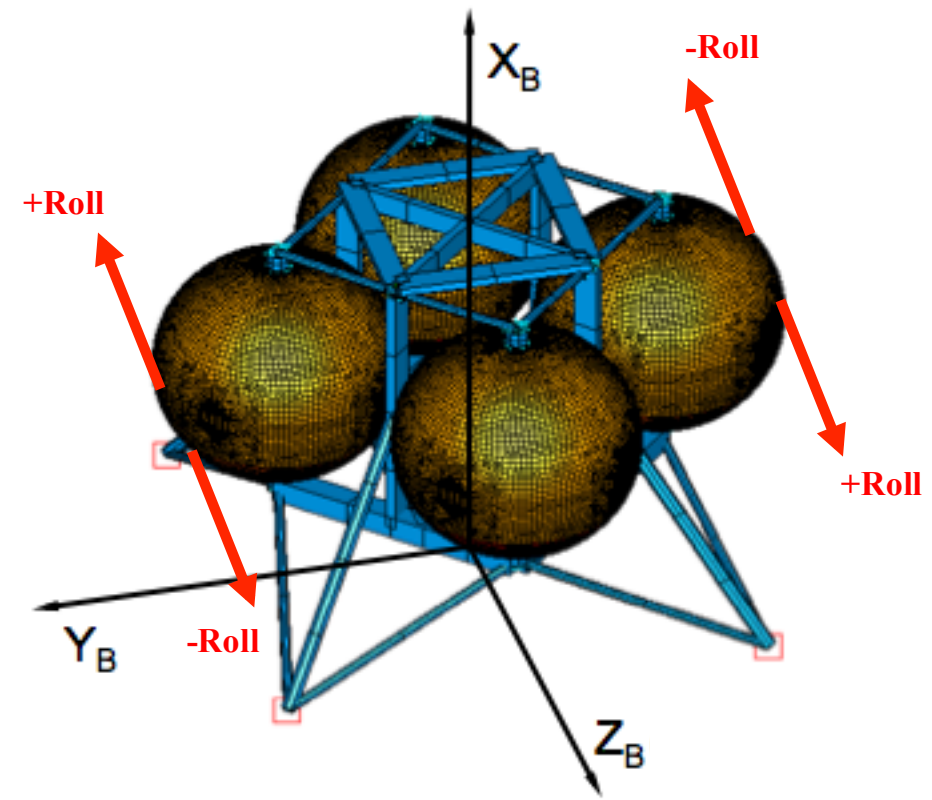

Figure 1. Morpheus roll control system.

The software algorithm controlling these thrusters consists of a phase plane that specifies roll and rate error limits within which the jets do not fire. The phase plane is tiered outside the limits to allow increasing firing requests. The firing requests include mandated delay times to finely control vehicle orientation. The tiered roll control phase plane is implemented as shown in Figure 2. 


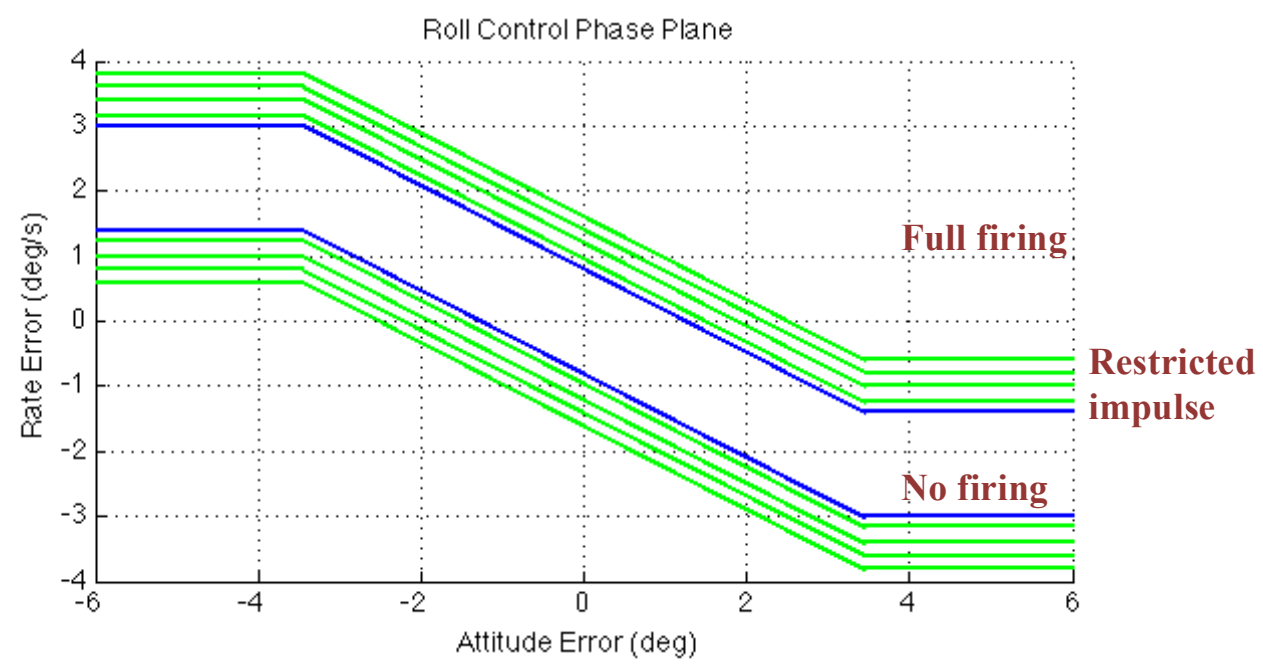

Figure 2. Morpheus roll control phase plane.

Vehicle testing demonstrated that roll torque depends greatly on wind speed and direction. As a result, a tool was developed that converts historical data and DOF wind conditions into wind profiles that are read by the simulation. The tool calculated average KSC wind conditions based on 13 years of wind tower data and generated monthly wind profiles. The tool was expanded to combine data from a 150 meter wind tower and high altitude Doppler radar at KSC to generate realistic DOF wind profiles for simulation analysis. Data tables were delivered the morning of each test for pre-flight certification. If the data sources did not match, the wind tower data could be extended using logarithmic curve fitting. This capability has allowed for the thorough testing of the Morpheus RCS using both historical winds and predicted flight conditions. The wind model can be further developed to create DOF profiles for various geographic locations. An illustration of the DOF wind profiles is shown in Figure 3.
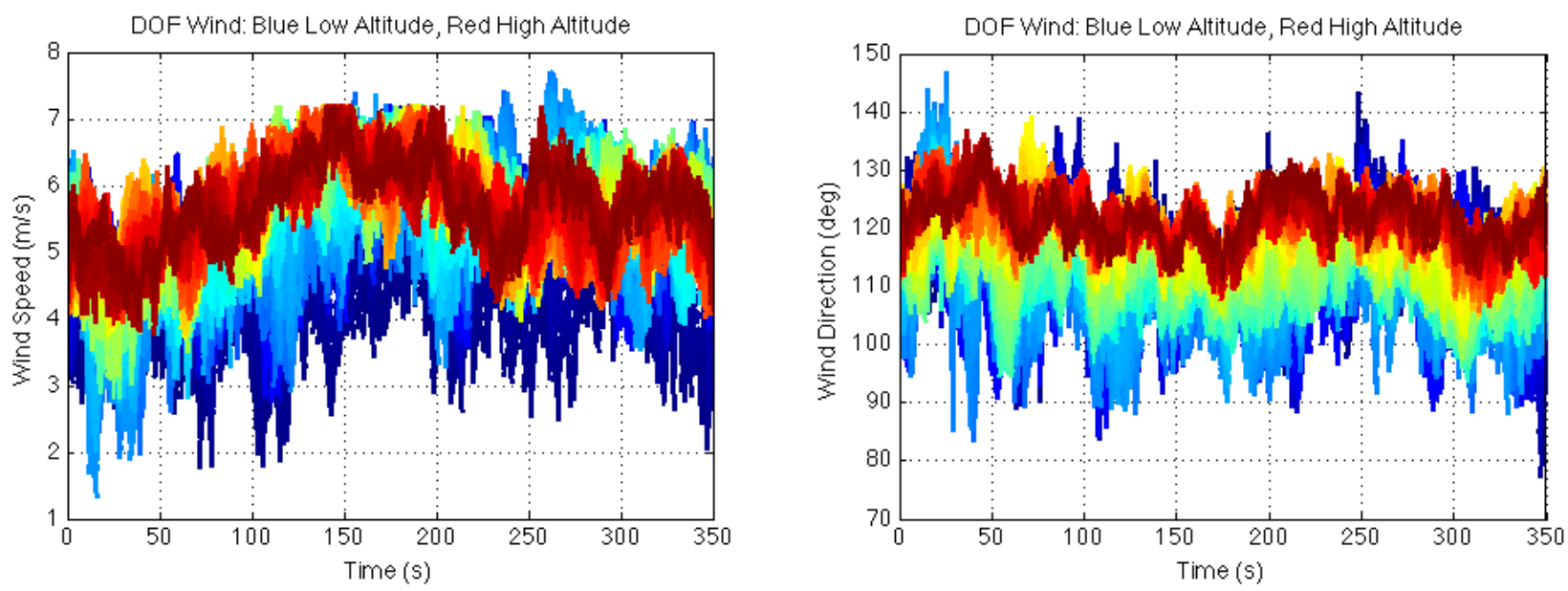

Figure 3. Sample Morpheus simulation wind profile.

The Morpheus vehicle has successfully flown trajectories reaching 250 meters altitude with wind conditions that verify the robustness of the RCS. Recent flight tests have demonstrated how the phase plane maintains roll orientation while preventing cycling between error limits. Further analysis will be completed to emphasize how the DOF wind profiles contributed to the successful completion of flight objectives. 


\title{
Morpheus Lander Roll Control System and Wind Modeling
}

\author{
Elisabeth A. Gambone ${ }^{1}$ \\ NASA Johnson Space Center, Houston, Texas, 77058
}

\begin{abstract}
The Morpheus prototype lander is a testbed capable of vertical takeoff and landing developed by NASA Johnson Space Center to assess advanced space technologies. Morpheus completed a series of flight tests at Kennedy Space Center to demonstrate autonomous landing and hazard avoidance for future exploration missions. As a prototype vehicle being tested in Earth's atmosphere, Morpheus requires a robust roll control system to counteract aerodynamic forces. This paper describes the control algorithm designed that commands jet firing and delay times based on roll orientation. Design, analysis, and testing are supported using a high fidelity, 6 degree-of-freedom simulation of vehicle dynamics. This paper also details the wind profiles generated using historical wind data, which are necessary to validate the roll control system in the simulation environment. In preparation for Morpheus testing, the wind model was expanded to create day-of-flight wind profiles based on data delivered by Kennedy Space Center. After the test campaign, a comparison of flight and simulation performance was completed to provide additional model validation.
\end{abstract}

\section{Nomenclature}

$\begin{array}{ll}I & =\text { moment of inertia } \\ t & =\text { time } \\ \tau & =\text { torque } \\ \omega & =\text { attitude rate }\end{array}$

\section{Introduction}

$\mathrm{T}$ HE Morpheus prototype lander was developed by engineers at NASA Johnson Space Center and serves as a testbed for autonomous landing and hazard avoidance technology. The vehicle is fueled with liquid oxygen and methane, a clean and non-toxic propellant with potential for future space missions. Morpheus is capable of high altitude flight tests with autonomous navigation to a safe landing site. Tests were performed between December 2013 and May 2014 at Kennedy Space Center (KSC) in Cape Canaveral, Florida at the northern end of the Shuttle Landing Facility. The vehicle's roll control system (RCS) was designed to handle the planned trajectories and expected wind conditions at KSC. In order to certify flight readiness prior to testing, day-of-flight (DOF) wind data tables were delivered to the Morpheus team, which were converted into wind profiles for the simulation environment. These wind profiles allowed the Morpheus team to assess vehicle performance with accurate aerodynamic forces.

\section{Roll Control Logic}

The Morpheus RCS consists of four thrusters fueled by the main engine propulsion tanks. Two thrusters in both the positive and negative roll direction maintain vehicle pointing during flight. The RCS counteracts disturbances and allows the hazard avoidance technology to scan the terrain and navigate to a safe landing location. The thrusters are oriented on the vehicle as shown in Fig. 1, with the plume direction illustrated.

The initial Morpheus RCS has cold gas jets that vent helium from the pressurized propulsion tanks to orient the vehicle. The software algorithm controlling these vents consists of a phase plane that specifies roll and rate error limits within which helium is not vented. If the rate error is greater than the phase plane limits, a positive firing is commanded to generate a

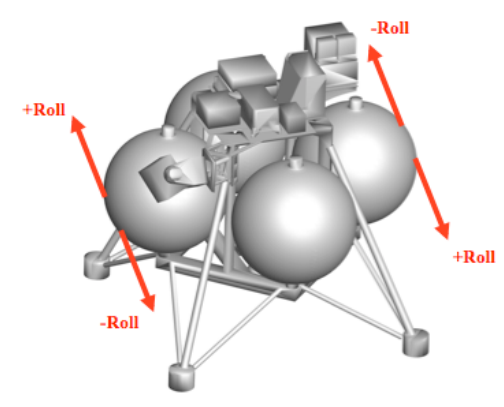

Figure 1. Morpheus roll control system.

\footnotetext{
${ }^{1}$ Aerospace Engineer; Integrated Guidance, Navigation, and Control Analysis; 2101 NASA Parkway, EG4 
counter-clockwise vehicle motion. Likewise, if the rate error is less than the phase plane limits, a negative firing is commanded. In the event of a large roll error, the phase plane includes drift channels that command a rate error in order to return the vehicle to zero error. This system proved ineffective during high altitude testing since tank pressurization directly corresponds to main engine thrust.

The methane jets provide nearly twice as much force as the helium vents, and the standard phase plane resulted in cycling between the error limits after a single thruster firing. A tiered phase plane was designed that constrains firing outside of the error limits to prevent cycling. The phase plane is implemented as shown in Fig. 2. The flight software is called such that a minimum impulse firing is 40 milliseconds. The firing requests increase as the rate error increases so the vehicle maintains the desired roll orientation. The firing requests include mandated delay times to prevent limit cycling. For instance, a 40 millisecond firing includes a delay time of 240 milliseconds to allow the vehicle to reposition itself before further firings are requested. If the rate error exceeds the restricted impulse region, the thrusters fire indefinitely to return the vehicle to the phase plane. The restricted impulse region, the on times, and the delay times can all be modified prior to flight if required. This RCS logic counteracts the roll torque created by vehicle motion and atmospheric conditions.

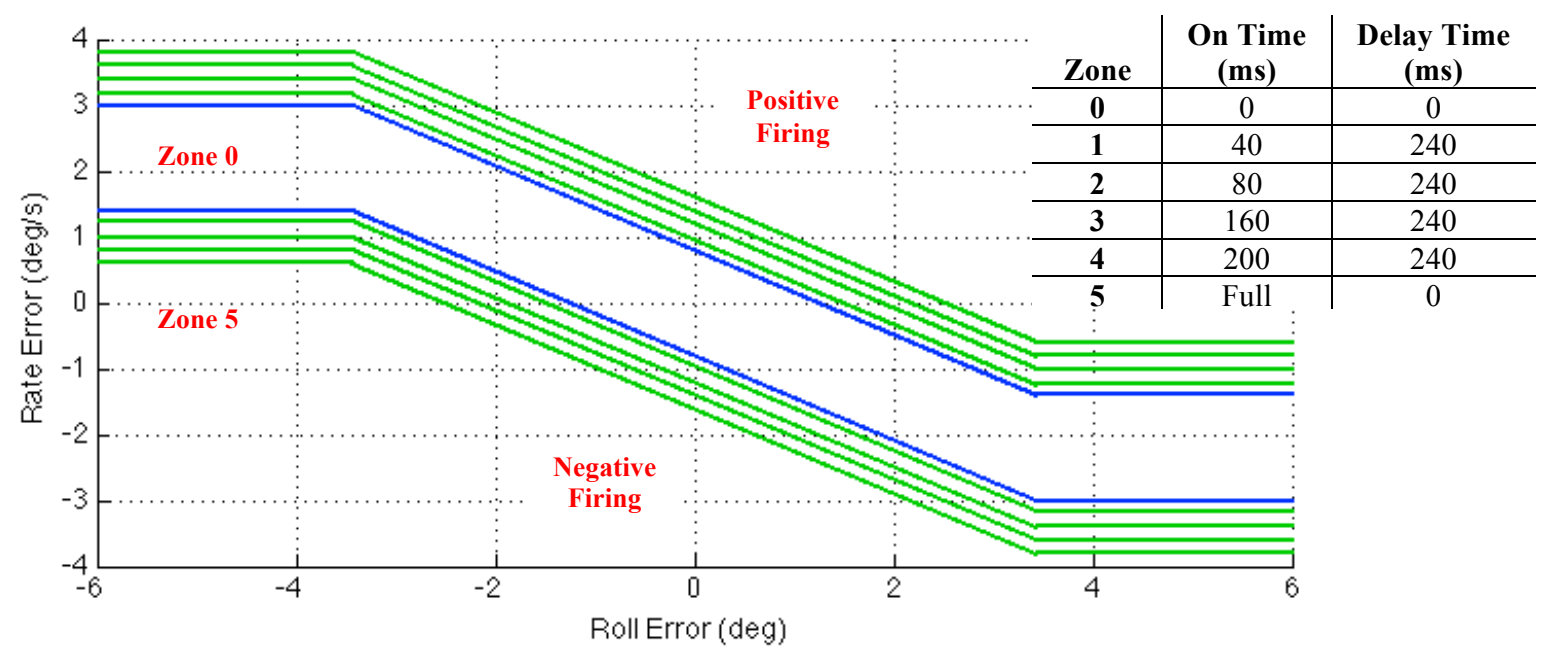

Figure 2. Morpheus roll control phase plane.

During Morpheus Free Flight 7 (FF7) on February 10, 2014, a negative RCS jet overheated after repetitive minimum impulse firing commands. The remaining thruster could not counteract the observed roll torque and return the vehicle to zero error, but the vehicle remained within two degrees roll error and the overheated jet eventually resumed operation. If FF7 had been performed on the initially planned date, the wind speed would have resulted in a disturbance torque that a single RCS thruster could not fight. This event led the Morpheus team to re-consider both the RCS design and the control logic. The phase plane tiers were modified to transition to longer firings at a smaller rate error in order to prevent jet overheating due to repetitive thruster ignitions. The analysis completed to determine the roll torque the RCS jets can withstand and the appropriate phase plane tiers relied heavily on the realistic wind model and aerodynamics implemented in the Morpheus simulation.

\section{Wind Modeling}

Vehicle testing confirmed that roll torque depends greatly on wind speed and direction. In extreme cases, the disturbance torque could exceed the capability of the vehicle RCS. As a result, a tool was developed that converts historical data and DOF wind conditions into wind profiles that are read by the simulation. This capability replaces a statistical wind model that does not capture the wind behavior at KSC and does not result in accurate RCS performance in the simulation. The natural environments group at Marshall Space Flight Center addressed this disparity by developing a turbulent wind profile generator that accepts wind tower data as its input. The Morpheus team created a tool that converts KSC wind data into the input format for the turbulent wind profile generator, creates a realistic KSC wind profile, and plots the generated profile. The output wind profile includes both a twodimensional wind speed and wind direction file, incrementing by simulation time and vehicle altitude. The wind profile is saved into the simulation so the wind condition corresponding to the vehicle state can be determined. An illustration of the DOF wind profiles is shown in Fig. 3, with each color representing a simulation time step. 

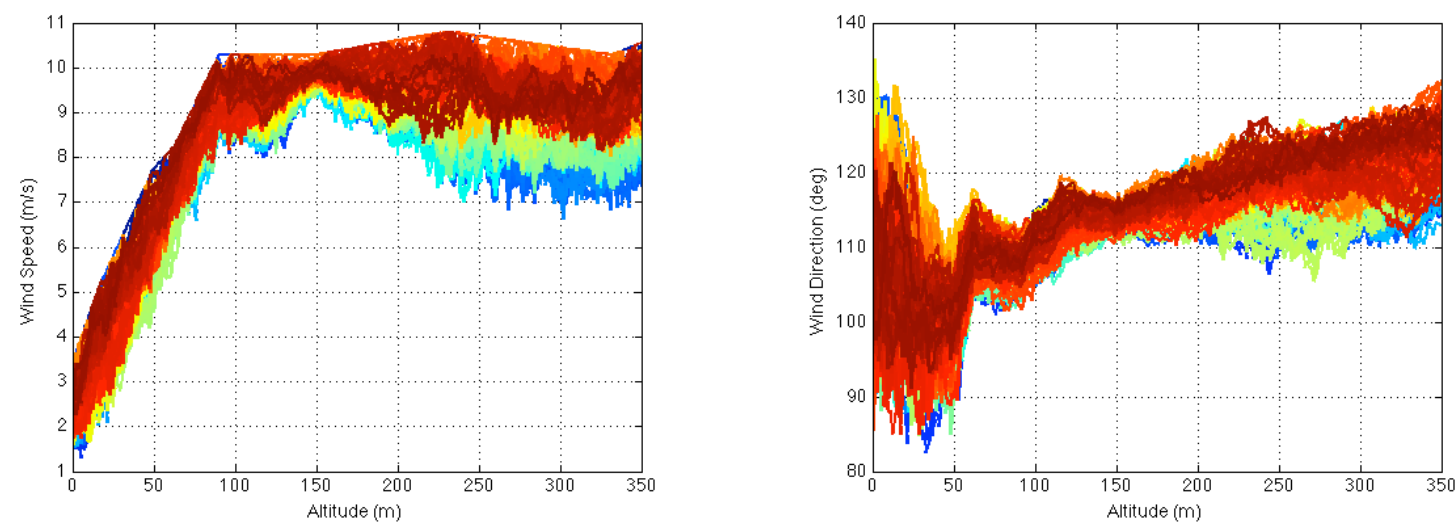

Figure 3. Day-of-flight wind profile.

The tool was used to calculate average KSC wind conditions based on 13 years of wind tower data and generate monthly wind profiles. These profiles were used to design test trajectories and verify RCS performance. The tool was expanded to combine data from a 150 meter wind tower and high altitude Doppler radar at KSC to generate realistic DOF wind profiles for simulation analysis. Data tables were delivered by the KSC meteorology group the morning of each test for pre-flight certification. The highest winds in each hourly wind tower data file were converted into the input for the turbulent profile generator. If the data sources did not match, the wind tower data could be extended using logarithmic curve fitting as shown in Fig. 4, which resembles the KSC wind behavior. This capability has allowed for the thorough testing of the Morpheus RCS using both historical winds and predicted flight conditions. The wind model can be further developed to create DOF profiles for other geographic locations.

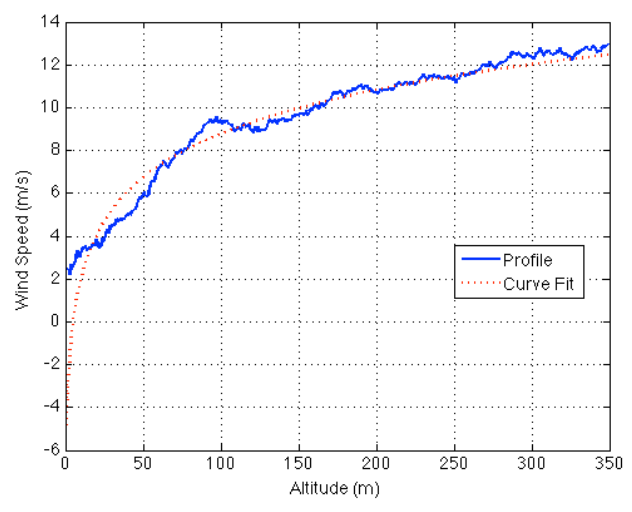

Figure 4. Curve fit wind profile.

\section{Vehicle Performance}

The DOF wind profiles were used to compare flight and simulation RCS performance and estimate the aerodynamic torques observed during testing. Analysis was completed on Morpheus FF13 flown on May 22, 2014. This test had 137 positive roll firing commands due to the wind direction and total airspeed on the vehicle during traverse. The vehicle reached an altitude of 246 meters, traversed downrange 405 meters, and landed on a pad within a lunar rock field. Winds reached approximately 6 meters per second blowing to the southeast. The flight versus simulation vehicle trajectory is shown in Fig. 5 and the simulated wind conditions are shown in Fig. 6. Launch and landing forces, propellant slosh, and vehicle vibration can cause dispersions in the flight trajectory.
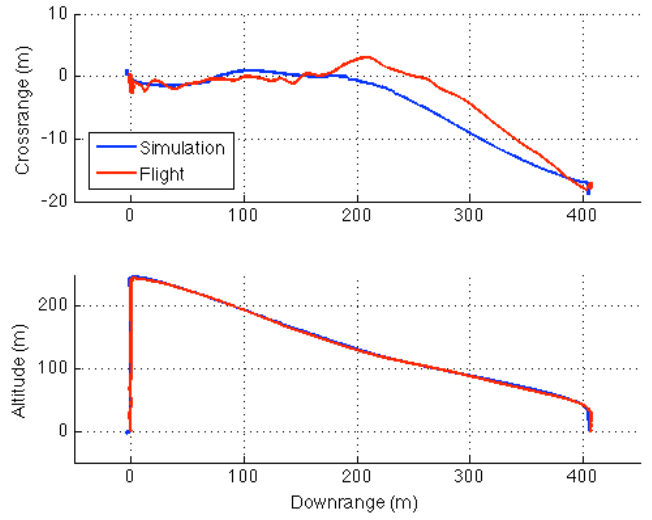

Figure 5. Morpheus flight versus simulation trajectory.
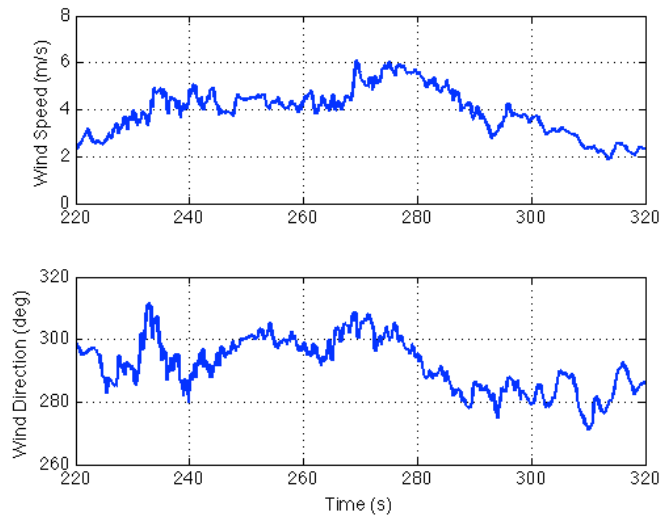

Figure 6. Simulation wind profile. 
The flight and simulation RCS firing commands are shown in Fig. 7. In the flight data, the vehicle ascended to maximum altitude between 12 and 38 seconds Mission Elapsed Time (MET). The vehicle then traversed downrange between 38 and 88 seconds MET. The vehicle finally descended to the landing pad between 88 and 106 seconds MET. In both the flight and simulation, rapid RCS firings occurred while the vehicle was traversing downrange at a speed of 11.8 meters per second. The wind and vehicle motion caused a total airspeed of 17.5 meters per second and increased the aerodynamic torque on the vehicle. The negative roll torque rotated the vehicle clockwise, and rapid RCS firings were commanded to counteract the motion. The flight and simulation phase plane are shown in Fig. 8. A zoom of the flight RCS commands and phase plane are also shown in Appendix A. As the roll error increased, the commanded RCS firings increased based on the phase plane tiers. The longest firings prior to landing were 160 milliseconds with a mandated 240 millisecond delay between firings. These commands kept the roll error within two degrees throughout the flight. The DOF wind profile allowed for the accurate representation of RCS performance in the simulation environment.
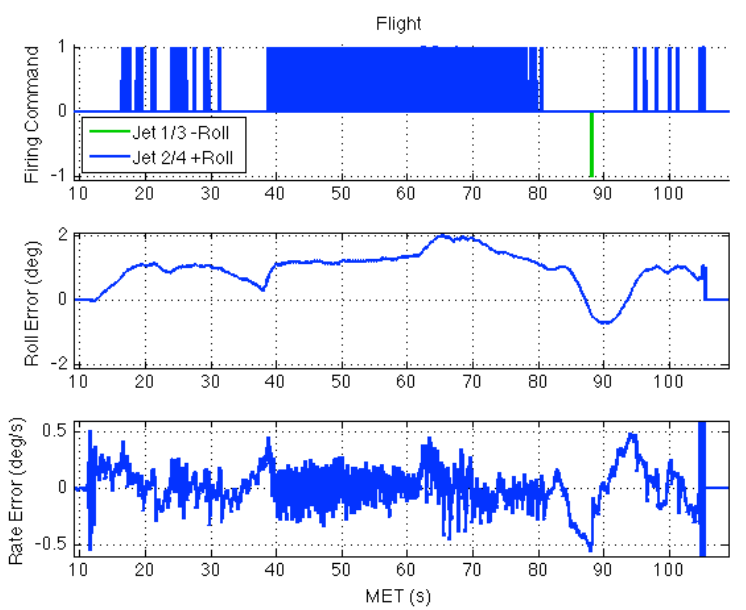
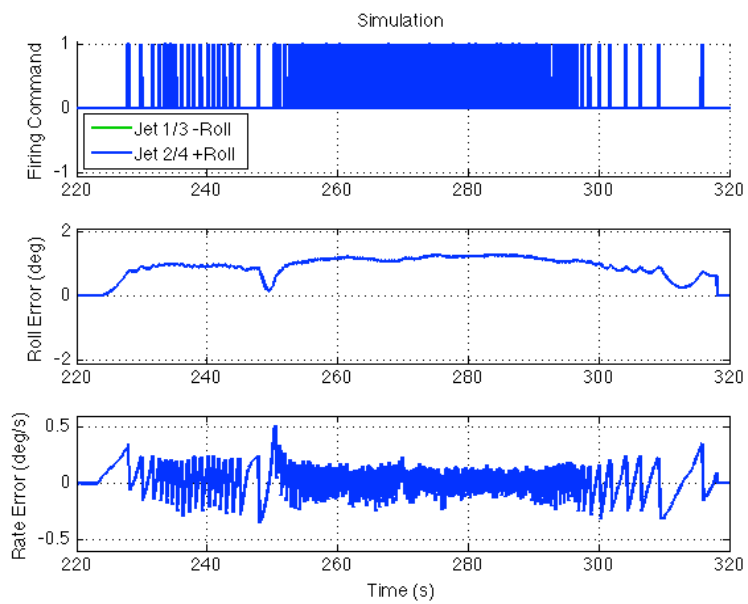

Figure 7. Morpheus flight versus simulation RCS firing commands.
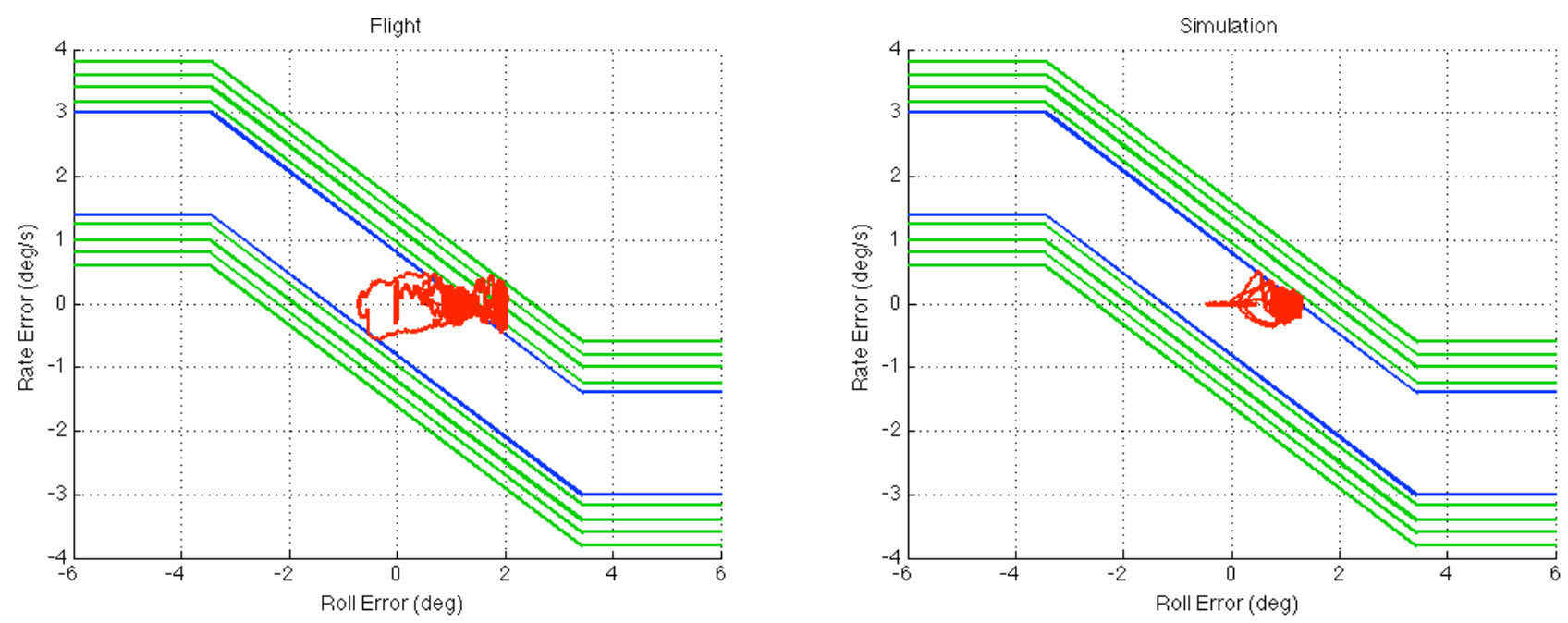

Figure 8. Morpheus flight versus simulation phase plane.

\section{Torque Estimation}

The torque $\tau$ generated by each RCS firing during FF13 was approximated using Eq. (1). The roll moment of inertia $I$, the change in roll rate error for each firing $\omega$, and the commanded time of each firing $t$ were deduced from the flight data. The approximated RCS and disturbance torques are shown in Fig. 9. The disturbance torque during flight corresponds to the roll torque generated in the simulation using the DOF wind profile. 


$$
\tau=I \frac{d \omega}{d t}
$$
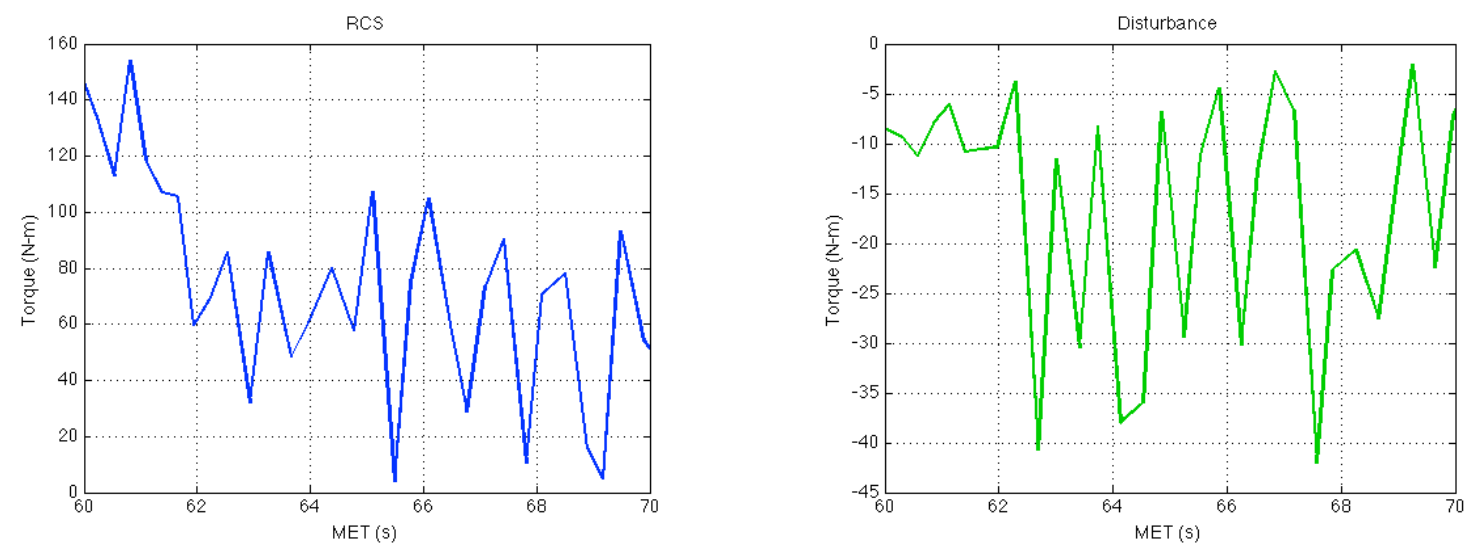

Figure 9. Approximated RCS torque and disturbance torque.

The simulation and wind model were utilized to determine the worst case wind conditions and corresponding roll torques. The FF13 trajectory was simulated with winds from varying directions, as shown in Fig. 10. The vehicle flew a northern trajectory, and a headwind resulted in the highest total airspeed. Likewise, a tailwind resulted in the lowest total airspeed. Due to the orientation of the vehicle during flight, both a headwind and tailwind resulted in less frequent RCS firings in both the positive and negative roll directions. A western crosswind created a large positive roll torque and rotated the vehicle counter-clockwise. This rotation resulted in frequent negative RCS firings to counteract the motion. Correspondingly, an eastern crosswind caused a large negative roll torque and rotated the vehicle clockwise. This negative rotation resulted in repetitive positive RCS firings, as seen in flight during FF13. Appendix B summarizes the maximum altitude, wind conditions, and RCS firings for recent Morpheus flight tests. The RCS firings can mainly be predicted by the airspeed heading, which is measured from the trajectory path. In earlier

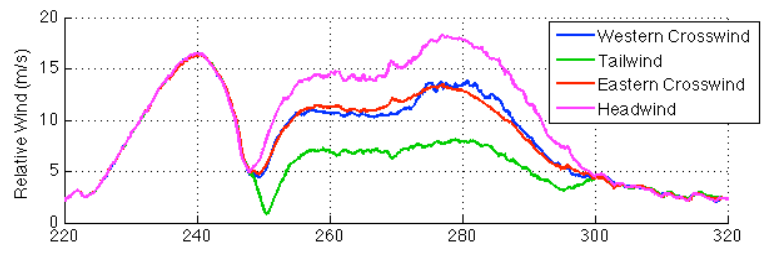
flights, the maximum altitude and vehicle velocity were lower, resulting in fewer RCS firings.

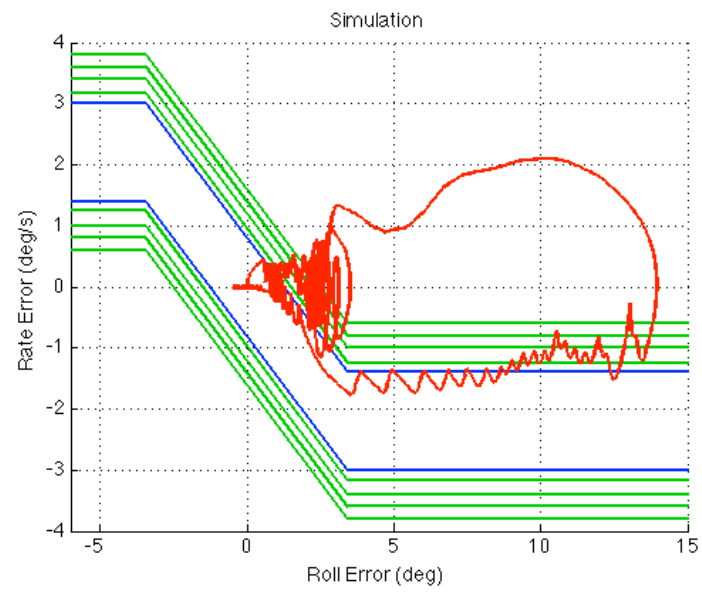

Figure 11. Vehicle response to high winds.

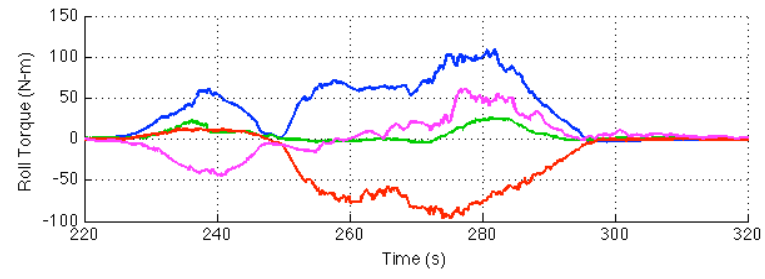

Figure 10. Wind direction versus roll torque.

The FF13 trajectory was additionally simulated with wind speeds upwards of 10 meters per second. At these wind speeds, the airspeed reached 20 meters per second, and the negative roll torque pushed the vehicle orientation outside the phase plane tiers, as shown in Fig. 11. The RCS thrusters fired longer pulses as the vehicle left the phase plane, eventually firing continuously to produce a negative roll rate and return the vehicle to the phase plane. The RCS jets overcame the roll torque and the vehicle landed safely, but this behavior is not desired in flight. Prior to modifying the RCS jets and phase plane, the thrusters could have overheated and been unable to counteract aerodynamic forces. An abort due to loss of roll control would cause the vehicle to land in untested terrain. Moreover, a loss of vehicle orientation could be catastrophic. As a result, a wind placard was proposed based on flight data and simulation analysis so the vehicle could be tested safely. 


\section{Conclusion}

The Morpheus vehicle has successfully flown trajectories reaching 246 meters altitude with wind conditions that verify the robustness of the RCS. Recent flight tests have demonstrated how the phase plane maintains roll orientation while preventing cycling between error limits. The phase plane tiers were exercised without a need for full thruster firings. The simulation wind model allowed operators to perform pre-flight certifications of vehicle performance with realistic wind conditions and roll torques. While work can be done to improve the accuracy of the simulation RCS model and vehicle vibration, the simulated RCS jets responded similarly to flight performance. The wind model proved extremely beneficial to the Morpheus team during vehicle development and testing.

\section{Appendix A}

\section{Morpheus FF13 RCS Performance}
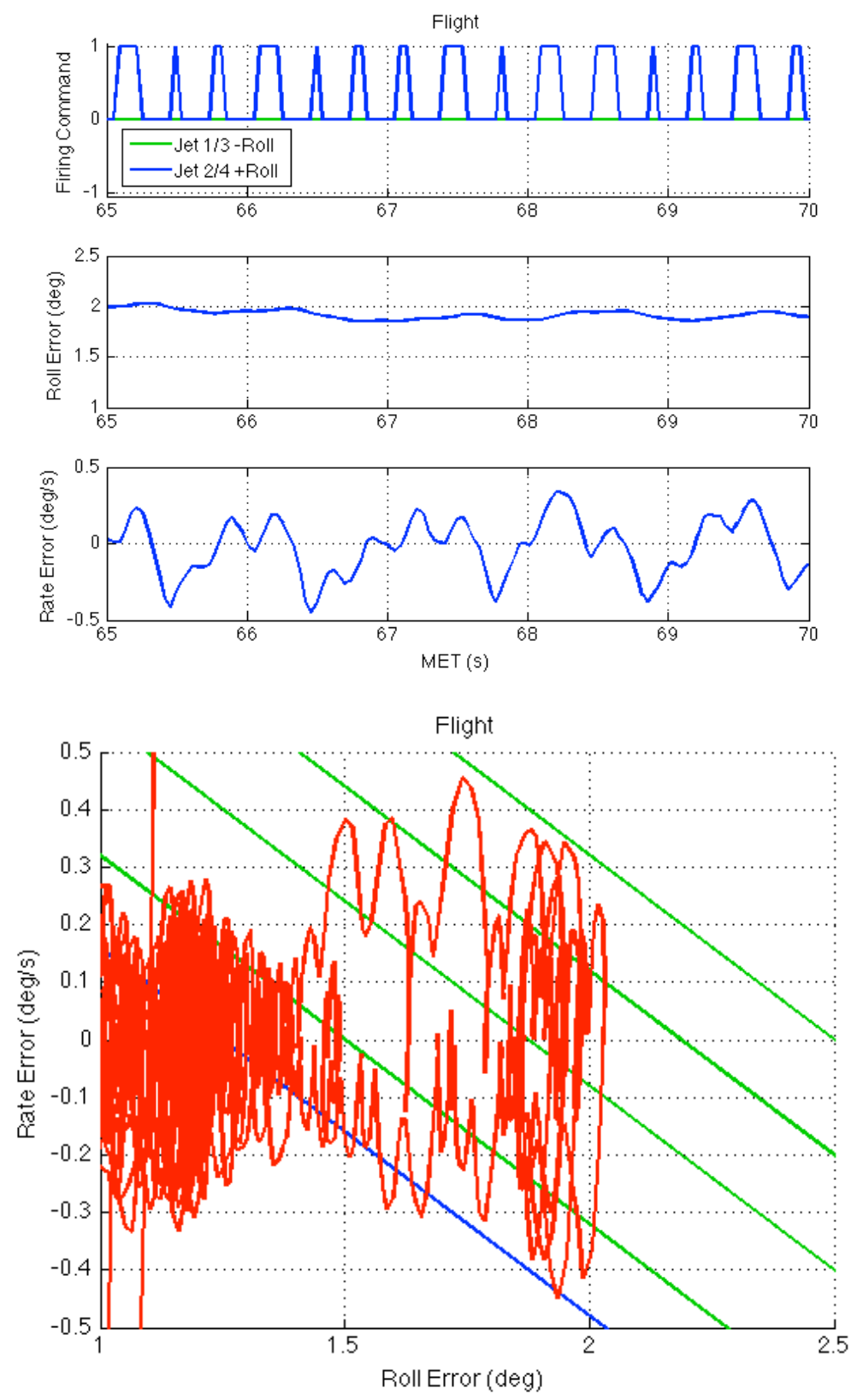

6

Morpheus Lander Roll Control System and Wind Modeling 


\section{Appendix B}

\section{Summary of Morpheus RCS Performance}

\begin{tabular}{c|c|c|c|c|c|c|c}
$\mathbf{F F}$ & $\begin{array}{c}\text { Altitude } \\
(\mathbf{m})\end{array}$ & $\begin{array}{c}\text { Velocity } \\
(\mathbf{m} / \mathbf{s})\end{array}$ & $\begin{array}{c}\text { Wind Speed } \\
(\mathbf{m} / \mathbf{s})\end{array}$ & $\begin{array}{c}\text { Airspeed } \\
(\mathbf{m} / \mathbf{s})\end{array}$ & $\begin{array}{c}\text { Airspeed Heading } \\
(\mathbf{d e g})\end{array}$ & $\begin{array}{c}+ \text { Roll } \\
\text { Firings }\end{array}$ & $\begin{array}{c}\text {-Roll } \\
\text { Firings }\end{array}$ \\
\hline 3 & 15 & 1.4 & 5.0 & 7.0 & 90 & 8 & 10 \\
\hline 4 & 50 & 2.8 & 6.5 & 11.1 & -15 & 7 & 21 \\
\hline 5 & 57 & 4.1 & 8.0 & 13.3 & 10 & 22 & 1 \\
\hline 6 & 93 & 7.8 & 5.5 & 11.0 & 100 & 9 & 18 \\
\hline 7 & 142 & 11.3 & 4.5 & 17.8 & -15 & 6 & 39 \\
\hline 8 & 142 & 8.4 & 8.0 & 17.3 & -10 & 11 & 54 \\
\hline 9 & 178 & 13.3 & 5.5 & 10.8 & -50 & 8 & 49 \\
\hline 10 & 246 & 11.8 & 6.0 & 9.5 & -40 & 13 & 22 \\
\hline 11 & 246 & 11.8 & 8.0 & 8.2 & -35 & 40 & 20 \\
\hline 12 & 246 & 11.8 & 9.0 & 7.3 & 0 & 57 & 17 \\
\hline $\mathbf{1 3}$ & $\mathbf{2 4 6}$ & $\mathbf{1 1 . 8}$ & $\mathbf{4 . 5}$ & $\mathbf{1 7 . 5}$ & $\mathbf{1 0}$ & $\mathbf{1 3 7}$ & $\mathbf{1}$ \\
\hline 14 & 246 & 11.8 & 6.0 & 6.9 & -10 & 21 & 20
\end{tabular}

\section{Acknowledgments}

The author would like to thank Jeremy Hart and Louis Nguyen for guidance and support during Morpheus roll control system and wind modeling tasks.

\section{References}

${ }^{1}$ Hart, J. J., and Devolites, J. L., “Morpheus Vertical Test Bed Flight Testing,” IEEE Aerospace Conference, 2014.

${ }^{2}$ Jang, J., Yang, L., and Fritz, M., "Design and Analysis of Morpheus Lander Flight Control System," AIAA/AAS Astrodynamics Specialist Conference, 2014.

${ }^{3}$ McManamen, J. P., Hurlbert, E.A, and Kroeger, D.J., "Development and Flight Operation of a $5 \mathrm{lb}_{\mathrm{f}}$ to $20 \mathrm{lb}_{\mathrm{f}} \mathrm{O}_{2} / \mathrm{CH}_{4} \mathrm{Roll}$ Control Engine for Project Morpheus,” AIAA/ASME/SAE/ASEE Joint Propulsion Conference, 2014. 